\title{
Minimally traumatic alveolar ridge augmentation with a tunnel injectable thermo-sensitive alginate scaffold
}

\author{
Yifen LI ${ }^{1,2 *}$, Xiaoqian FANG ${ }^{1 *}$, Ting JIANG ${ }^{1}$ \\ 1- Department of Prosthodontics, Peking University School and Hospital of Stomatology, Beijing, China; Department of Prosthodontics, Jiangmen Municipal \\ Stomatological Hospital, Jiangmen, Guangdong, China. \\ 2- Department of Prosthodontics, Peking University School and Hospital of Stomatology, Beijing, China. \\ *These authors contributed to the work equally and should be regarded as co-first authors.
}

Corresponding address: Ting Jiang - Department of Prosthodontics - Peking University School and Hospital of Stomatology, 22 Zhongguancun Avenue South - Haidian District - Beijing 100081 - China - Phone: 010-82195348 - 15011229485 - e-mail: jt ketizu@163.com

Submitted: November 28, 2014 - Modification: January 29, 2015 - Accepted: March 2, 2015

\section{ABSTRACT}

\begin{abstract}
Tnjectable bone substitutes and techniques have been developed for use in minimally 1 invasive procedures for bone augmentation. Objective: To develop a novel injectable thermo-sensitive alginate hydrogel (TSAH) as a scaffold to induce bone regeneration, using a minimally invasive tunnelling technique. Material and Methods: An injectable TSAH was prepared from a copolymer solution of $8.0 \mathrm{wt} \%$ Poly( $\mathrm{N}$-isopropylacrylamide) (PNIPAAm) and $8.0 \mathrm{wt} \%$ AAlg-g-PNIPAAm. In vitro properties of the material, such as its microstructure and the sustained release of recombinant human bone morphogenetic protein-2 (rhBMP-2), were investigated. Then, with the subperiosteal tunnelling technique, this material, carrying rhBMP-2, was injected under the labial periosteum of the maxillary anterior alveolar ridge in a rabbit model. New bone formation was evaluated by means of $\mathrm{X}$-ray, micro-computed tomography (micro-CT), fluorescence labelling, histological study, and immunohistochemistry study. Results: The material exhibited good injectability and thermo-irreversible properties. SEM showed an interconnected porous microstructure of the TSAH. The result of ALP activity indicated sustained delivery of BMP-2 from the TSAH from days 3 to 15. In a rabbit model, both TSAH and TSAH/rhBMP-2 induced alveolar ridge augmentation. The percentage of mineralised tissue in the TSAH/rhBMP-2 group $(41.6 \pm 3.79 \%)$ was significantly higher than in the TSAH group $(31.3 \pm 7.21 \% ; \mathrm{p}<0.05)$. The density of the regenerating tissue was higher in the TSAH/rhBMP-2 group than in the other groups (TSAH group, positive control, blank control; $p<0.05$ ). Conclusions: The TSAH provided convenient handling properties for clinical application. To some extent, TSAH could induce ridge augmentation and mineral deposition, which can be enhanced when combined with rhBMP-2 for a minimally invasive tunnelling injection.
\end{abstract}

Keywords: Alveolar ridge augmentation. Minimally invasive surgical procedures. Hydrogel. Tissue engineering.

\section{INTRODUCTION}

Atrophic maxillary anterior alveolar ridge fails to provide sufficient support or aesthetics for dental prostheses ${ }^{24}$. Today, allografts and xenograft bone grafts are the major methods for augmenting a resorbed alveolar ridge. However, they have several disadvantages, such as the risk of disease transmission and immunological reactions ${ }^{3}$. Another disadvantage is that these grafts require an open surgical operation ${ }^{8}$. To reduce trauma and surgery cost, "injectable tissue-engineered bone" has been proposed. Injectable systems - materials and techniques - are being developed for use in minimally invasive procedures and their ability to fill irregular defects ${ }^{1,7}$.

In the late 1980 s, Kent, et al. ${ }^{12,13}(1982,1983)$ proposed the sub-periosteal tunnelling technique. 
They made a small incision in the mucosa of the alveolar ridge, elevated the periosteal, and injected hydroxyapatite particles into the tunnel through a modified syringe. Although the material diffused into adjacent tissues, leading to poor bone regeneration, the technique attracted attention. However, due to the limitations of the injectable bone substitute material, this technique did not become popular clinically.

Injectable biomaterials now include synthetic ceramics and synthetic polymers ${ }^{16}$. In recent years, thermo-sensitive hydrogel systems have attracted much interest in the biomedical field ${ }^{22}$. Poly(N-isopropylacrylamide) (PNIPAAm) is one such synthetic polymer that can form a hydrogel in situ above its lower critical solution temperature (LCST) and maintain a liquid state below the LCST. Alginate is a natural polysaccharide composed of 1,4 -linked $\beta$-D-mannuronate and 1,4-linked a-L-guluronate residues. It is extracted from algin, which is abundant and inexpensive. It also has the advantages of good biocompatibility and immunological non-responsiveness ${ }^{20}$. The AAlg-g-PNIPAAm copolymer, prepared through coupling carboxylic end-capped PNIPAAm to aminated alginate (AAlg), has good injectability and plasticity at room temperature, while it gels at body temperature $\left(37^{\circ} \mathrm{C}\right)$. In particular, it has been shown to be non-cytotoxic and can promote the proliferation of entrapped cells. The degradation rate is controllable by changing the quantity of PNIPAAm ${ }^{23}$. By adding 8.0 wt\% PNIPAAm to 8.0 wt\% AAlg-g-PNIPAAm copolymer, we obtained a TSAH that seemed to be a suitable delivery system for minimally invasive bone regeneration.

Bone morphogenetic protein-2 (BMP-2), one of the most promising osteoinductive growth factors, can recruit progenitor cells and promote the differentiation of osteoblasts ${ }^{2,25}$. Because of the short half-life and initial burst release of BMP-2, biocompatible carriers such as the thermo-sensitive alginate are necessary to protect its bioactivity and prolong the release period ${ }^{15}$.

In this study, we combined the TSAH with BMP2 to explore the effect of this delivery system on bone augmentation of the anterior alveolar ridge in a rabbit model, using the sub-periosteal tunnelling technique. The micro-structure of the TSAH and the sustained release properties of rhBMP-2 from the TSAH in vitro were also explored.

\section{MATERIAL AND METHODS}

\section{Preparation of the TSAH}

An AAlg-g-PNIPAAm copolymer with $29 \%$ PNIPAAm grafting was prepared. The copolymer was synthesised by grafting PNIPAAM-COOH with a single carboxyl end group onto aminated alginate (AAlg) through amide bond linkages ${ }^{23}$. Dry PNIPAAm and AAlg-g-PNIPAAm were sterilised with an electron beam, dissolved in phosphate buffer solution (PBS, $\mathrm{pH} 7.4$ ), and stirred overnight at $4^{\circ} \mathrm{C}$. In order to obtain an optimum mixture ratio, different percentages of PNIPAAm (6.0, $8.0 \mathrm{wt} \%$ ) were mixed into different percentages of AAlg-gPNIPAAm (8.0, 10.0, 12.0 wt\%). We detected the injectability and the hardness after gelation of the materials at each mixture ratio. Finally, a delivery system containing 8.0 wt $\%$ PNIPAAm and 8.0 wt\% AAlg-g-PNIPAAm was chosen and prepared.

\section{Morphological observation}

The TSAH was first gelled at $37^{\circ} \mathrm{C}$, lyophilised into a dry sample, and then fractured. The crosssection was gold-coated and the inner morphology of the TSAH was observed by scanning electron microscopy (SEM, Hitachi S-4800, Japan) at $15 \mathrm{kV}$.

\section{Bioactivity of rhBMP-2 released from the hydrogel in vitro \\ The recombinant human BMP-2 (rhBMP-2;} PeproTech, USA) was dissolved in PBS containing bovine serum albumin (BSA) $(1 \mu \mathrm{g}$ of rhBMP-2 per $50 \mu \mathrm{g}$ of $\mathrm{BSA})$, then the solution was mixed into the TSAH. The final BMP-2 concentration was 50 $\mu \mathrm{g} / \mathrm{mL}$. All preparations were carried out under aseptic conditions.

TSAH/rhBMP-2 $(250 \mu \mathrm{L})$ and TSAH samples (250 $\mu \mathrm{L})$ were injected separately into $1.5-\mathrm{mL}$ capped centrifuge tubes and incubated at $37^{\circ} \mathrm{C}$ for a few minutes for gelation. Then, $1 \mathrm{~mL}$ of PBS was added into each tube and incubated in a rotary shaker (35 $\mathrm{rpm}$ ) at $37^{\circ} \mathrm{C}$ for 21 days. At scheduled intervals $(1,3,5,7,9,11,15,18$ and 21 days), $0.5 \mathrm{~mL}$ of supernatant were collected and replaced with 0.5 $\mathrm{mL}$ of fresh PBS. The collected supernatant was sterilised by filtration and stored at $-20^{\circ} \mathrm{C}$ for later use.

Human bone marrow-derived mesenchymal stem cells (hBMSCs) were plated at a density of $2 \times 10^{4}$ cells per well in 24 -well plates. Subsequently, the cells were incubated in a-MEM with $10 \%$ fetal bovine serum (FBS), $100 \mathrm{U} / \mathrm{mL}$ penicillin, and 100 $\mu \mathrm{g} / \mathrm{mL}$ streptomycin in a humidified $37^{\circ} \mathrm{C} / 5 \% \mathrm{CO}_{2}$ incubator. After $24 \mathrm{~h}$, the medium was replaced with $1 \mathrm{~mL}$ of fresh medium composed of obtained supernatant and a-MEM at a ratio of $1: 7(\mathrm{v} / \mathrm{v})$. The obtained supernatant without rhBMP-2 was used in the negative control group while $400 \mathrm{ng} / \mathrm{mL}$ rhBMP-2 were used in the positive control group. After 5 days in culture, the medium was removed, the cells were washed twice with PBS, and lysed with $0.5 \mathrm{~mL}$ of $1 \%$ Triton $\mathrm{X}-100$ combined with ultrasonication.

Alkaline phosphatase (ALP) is one of the most commonly used osteogenic markers. It is 
assumed to reflect the degree of the osteogenic differentiation ${ }^{18,26}$. It is also well-known that BMP-2 can increase the ALP activity of hBMSCs in a dosedependent manner. Thus, the amount and activity of BMP-2 released from the gel can be evaluated indirectly according to an ALP activity assay in vitro. The cell lysates were centrifuged and the supernatant was collected for ALP analysis with an ALP activity assay kit (Jiancheng, Nanjing, Jiangsu, China) according to the manufacturer's protocol.

\section{Animal experiments}

\section{In vivo bone augmentation}

In total, sixteen male New Zealand White rabbits, weighing $\sim 2.5 \mathrm{~kg}$, were allowed to acclimatize for 1 week in the laboratory animal housing prior to the operation. All experiment protocols were approved by the Biomedical Ethics Committees. Under general anaesthesia, through the otomarginal vein with pentobarbital sodium (30 $\mathrm{mg} / \mathrm{kg}$ ), an incision of $\sim 1 \mathrm{~mm}$ was made in the mucoperiosteum on the labial side of the maxillary anterior alveolar bone. A subperiosteal space with dimensions of $10 \times 5 \times 4 \mathrm{~mm}$ was created with a periosteal elevator. Afterwards, the material was injected through a syringe needle of $1.2 \mathrm{~mm}$ in diameter into the tunnel to occupy the space created and to augment the alveolar ridge. The incision was then closed with a suture (Figure 1A-D).

A total of 32 alveolar augmentation implants were injected bilaterally in 16 rabbits, divided randomly into the following four groups: A- TSAH ( $n=12,4$ for each time point: weeks 4, 8, 12); BTSAH/rhBMP-2 ( $n=12,4$ for each time point: weeks $4,8,12)$; C- injectable calcium phosphate/BMP-2 as a positive control $(n=4$, week 12$)$, and D- PBS as a blank control ( $n=4$, week 12$)$.

All rabbits were given antibiotics via a subcutaneous injection 3 days after the operation and were observed grossly every day. Animals were euthanised at 4, 8, and 12 weeks after surgery. Alveolar bone samples were harvested and fixed in $10 \%$ neutral buffered formalin. All specimens were analysed by radiography and histologically.

Radiography analyses and micro-CT assessment

The gross samples were examined using a dental X-ray machine (Warning, Finland) at 60 $\mathrm{kV}, 8 \mathrm{~mA}$ for $0.125 \mathrm{~s}$. Then, they were scanned by micro-computed tomography (micro-CT) (Siemens Inveon, Germany) at $60 \mathrm{kV}, 400 \mu \mathrm{A}$ in high resolution scanning mode to determine the bone volume. Bone mineral density (BMD) and bone volume fraction (bone volume/total volume, BV/TV) were also determined.

\section{Sequential fluorescent labelling}

To evaluate the distribution and rate of new bone formation, the rabbits sacrificed at 12 weeks post-surgery were labelled with three polychrome sequential fluorescent markers. At 3, 6 and 9 weeks after the surgical operation, the rabbits were injected subcutaneously at the implant sites with $60 \mathrm{mg} / \mathrm{kg}$ tetracycline hydrochloride (TE) (Sigma, St Louis, MO, USA), $30 \mathrm{mg} / \mathrm{kg}$ alizarin red (AL) (Sigma, St Louis, MO, USA), and $10 \mathrm{mg} / \mathrm{kg}$ calcein (CA) (Sigma, St Louis, MO, USA), respectively.

\section{Histological and histomorphometric} observations

The samples harvested at 4 and 8 weeks were decalcified completely in 20\% EDTA ( $\mathrm{pH}=7.2)$, embedded in paraffin wax, and sectioned with a microtome (Leica RM2235, Germany) to a thickness of $4 \mu \mathrm{m}$. Then, haematoxylin and eosin (HE) staining was performed and the expression of two osteoblast differentiation markers, osteopontin (OPN) and osteocalcin (OCN), was examined by
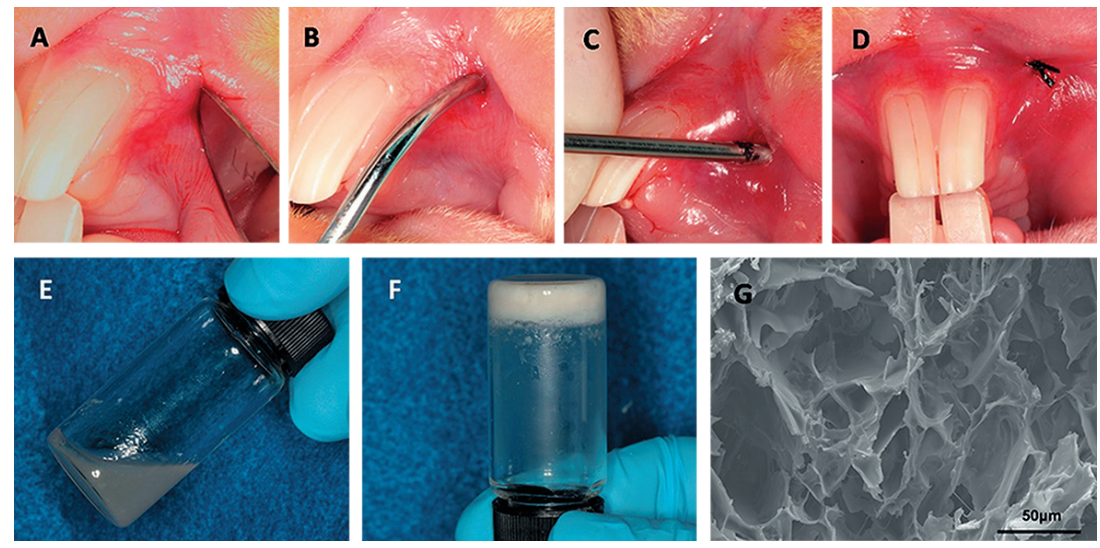

Figure 1- The subperiosteal tunnelling technique, sol-gel transformation and microstructure of the thermo-sensitive alginate hydrogel (TSAH). An incision was made on the mucoperiosteal surface (A), and a subperiosteal space was created (B). The material was injected into the tunnel to occupy the space created (C), and the incision was subsequently closed (D). The TSAH was flowable at room temperature $(E)$, but gelled above the lower critical solution temperature $\left(37^{\circ} \mathrm{C}\right)(\mathrm{F})$. The microstructure of the TSAH $(G)$ showed an interconnected porous microstructure, like a honeycomb 
immunohistochemical staining. Those harvested at 12 weeks post-surgery were bisected. Half of the gross samples was decalcified and stained as above. The remaining half was dehydrated, embedded in polymethylmethacrylate and cut into $150-\mu m$-thick sections with a microtome (EXAKT $300 \mathrm{CP}$, Germany). Finally the sections were ground and polished to a thickness of $40 \mu \mathrm{m}$ and observed under a fluorescence microscope.

\section{Statistical analysis}

All data are presented as means \pm standard deviations. Significant differences in in vitro bone formation analyses were analysed by one-way ANOVA. The differences between groups and time points were considered statistically significant if $\mathrm{p}<0.05$.

\section{RESULTS}

\section{Preparation of the TSAH}

The TSAH was flowable and could be injected smoothly through a needle of $1.2 \mathrm{~mm}$ in diameter at room temperature. Above the LCST $\left(37^{\circ} \mathrm{C}\right)$, it gelled and gained enough strength to support the periosteum (Figure 1E-F).

\section{Morphological observation}

The microstructure of the lyophilised TSAH was observed by scanning electron microscopy (SEM); the image is shown in Figure $1 \mathrm{G}$. The hydrogel presented an interconnected porous microstructure, like a honeycomb. Interconnected pores with sizes in the range of $30-50 \mathrm{~nm}$ could be found in the
TSAH.

\section{Bioactivity of rhBMP-2 released from the} hydrogel in vitro

The release profile of rhBMP-2 loaded in hydrogel is shown in Figure 2. ALP activity increased greatly at day 3 , indicating that osteoblast differentiation was induced by the initial exposure to BMP-2, and then maintained until day 15 . It was significantly higher than that in the negative control group $(p<0.05)$. From day 18 to day 21 , there was no obvious difference between the test group and control group. These results indicated that sustained delivery of BMP-2 from the gel stimulated ALP activity in hBMSCs from day 3 to day 15; however, the dose of BMP-2 released might be too low to affect ALP activity before and after this period.

\section{In vivo bone formation \\ Radiographic examination}

Radiographs were taken at 4, 8, and 12 weeks post-surgery for qualitative assessments of bone formation (Figure 3). There was no visible new bone formation in the samples at 4 and 8 weeks post-surgery in the TSAH and TSAH/rhBMP-2 groups but augmented images above the surface of alveolar ridge can be seen. At 12 weeks, some distributed high density areas were seen within the material. Furthermore, the radiopaque areas in the TSAH/rhBMP-2 group were larger than those of the TSAH group. The group filled with calcium phosphate cement (CPC)/rhBMP-2 showed no new bone formation. Interestingly, high-density spots were distinguishable on the buccal side of the

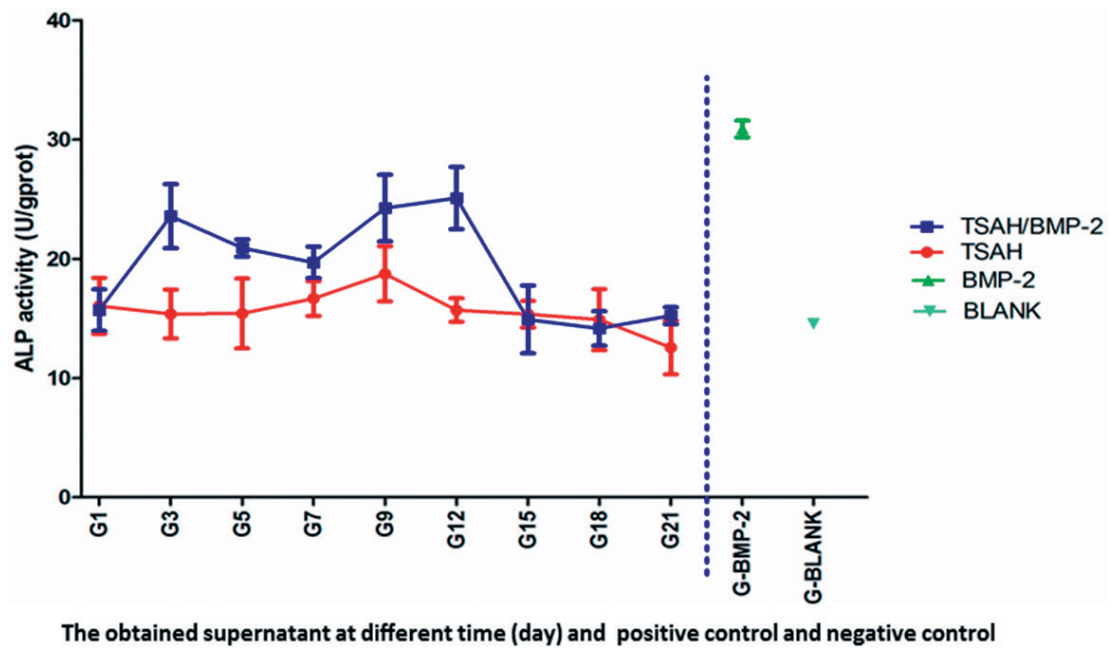

Figure 2- The bioactivity of the recombinant human bone morphogenetic protein-2 (rhBMP-2) released from the thermosensitive alginate hydrogel (TSAH) at different time points was evaluated by the alkaline phosphatase (ALP) activity of human bone marrow-derived mesenchymal stem cells (hBMSCs). The blue dots represent the ALP activity of hBMSCs cultured in the supernatant of TSAH/rhBMP-2 of different days. The red dots represent the ALP activity of hBMSCs cultured in the supernatant of TSAH of different days. The dark green dot represents the ALP activity of hBMSCs cultured with $400 \mathrm{ng} / \mathrm{mL}$ rhBMP-2 as positive control. The light green dot represents the ALP activity of hBMSCs cultured with fresh phosphate buffer solution (PBS) as negative control 

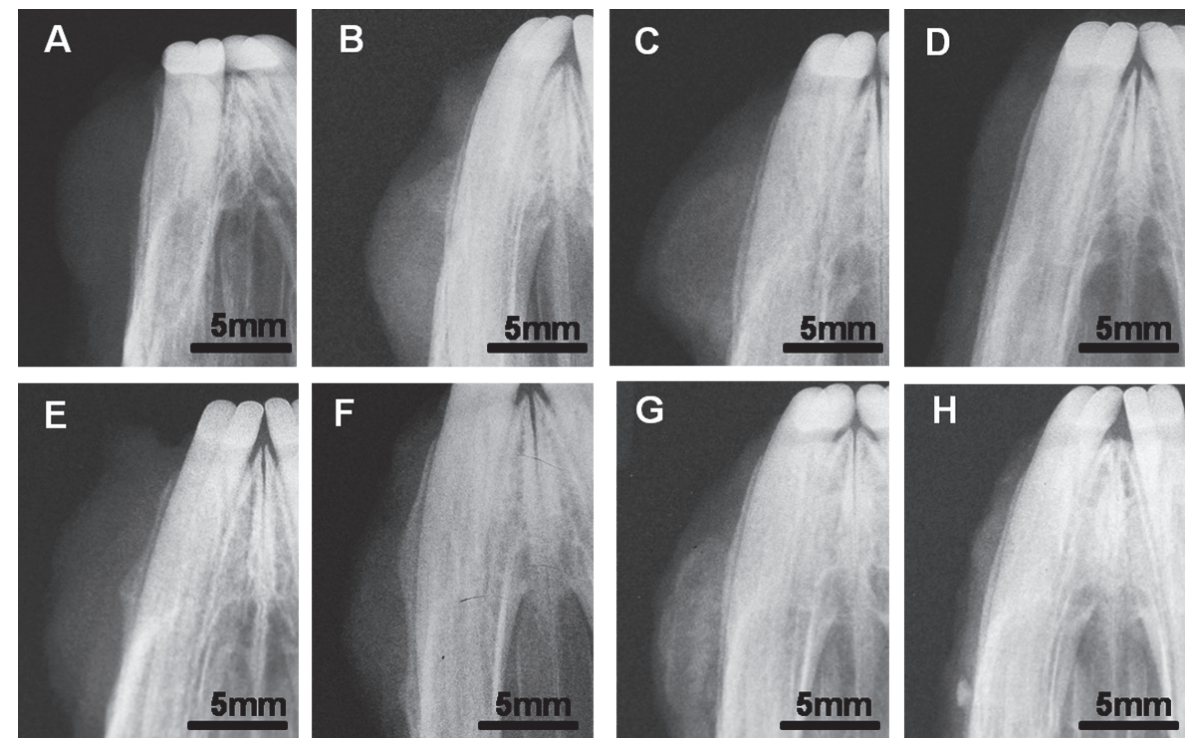

Figure 3- Radiographs of samples in the thermo-sensitive alginate hydrogel (TSAH) group at weeks 4, 8, and 12 (A, B, C, respectively), phosphate buffer solution (PBS) negative control group at week 12 (D), TSAH/bone morphogenetic protein-2 (BMP-2) group at weeks 4, 8, and 12 (E, F, G, respectively), and the calcium phosphate cement (CPC)/BMP-2 control group at week $12(\mathrm{H})$
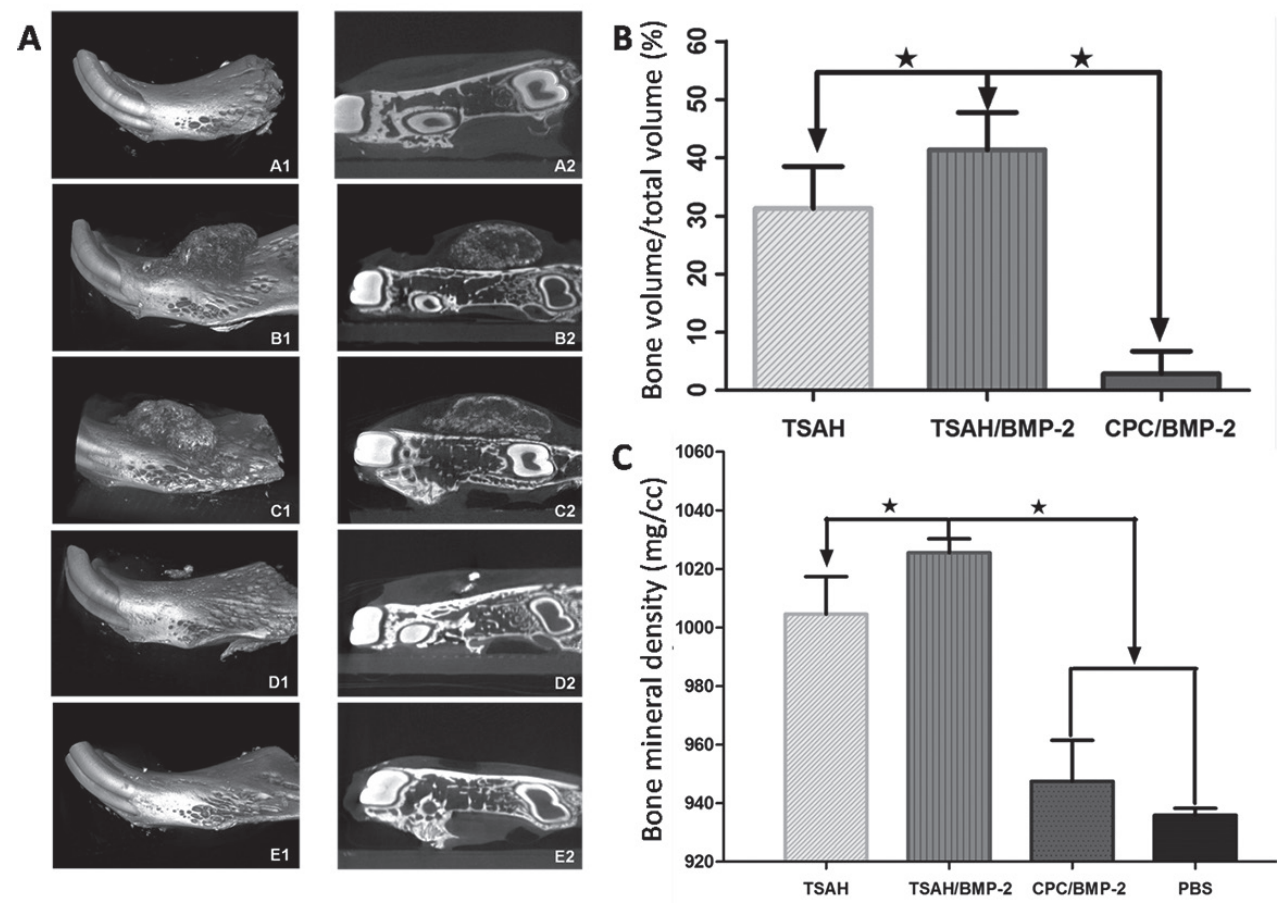

Figure 4- A- Representative $3 D$ micro-computed tomography reconstruction images (A1-E1) and sectional images (A2-E2) of the various groups. The immediate postoperative image of the thermo-sensitive alginate hydrogel (TSAH) was radiolucent $(\mathrm{A} 1, \mathrm{~A} 2)$. Bone augmentation was shown in the TSAH (B1, B2) and TSAH/recombinant human bone morphogenetic protein-2 (rhBMP-2) groups (C1, C2) after 12 weeks, with a high-density spot in the calcium phosphate cement (CPC)/rhBMP-2 group (D1, D2), and no effect in the phosphate buffer solution (PBS) group (E1, E2) at 12 weeks. B- The bone volume/total volume (BV/TV) in various groups. The thermo-sensitive alginate hydrogel/recombinant human bone morphogenetic protein-2 (TSAH/rhBMP-2) group had significantly more bone formation than the other groups ( $\star$ indicates significant difference, $p<0.05)$. C- The density of the regenerating tissue was higher in the TSAH/rhBMP-2 group than in the other groups ( $\star$ indicates significant difference, $p<0.05$ ) 
alveolar ridge, $10 \mathrm{~mm}$ from the injecting area in two samples. There was no bone augmentation in the PBS group.

\section{Micro-CT examination}

All of the harvested samples were scanned and reconstructed with a micro-CT system for quantitative assessment of bone formation (Figure 4A). The images showed successful bone augmentation on bone surfaces in the TSAH groups with or without rhBMP-2. At the periphery of the protuberant area, the amount of new bone formation was higher than that in the centre. The analysis of regenerated bone volumes also indicated that alveolar ridges were augmented $(\sim 10 \times 5 \times 4 \mathrm{~mm})$ in both the TSAH and the TSAH/ rhBMP-2 groups, whereas there were only high density spots $(\sim 1 \times 2 \times 2 \mathrm{~mm})$ in the CPC/rhBMP-2 group, and nothing in the PBS group at week 12 . The percentage of mineralised tissue in the TSAH/ rhBMP-2 group (41.6 $\pm 3.79 \%)$ was significantly higher than that in the TSAH group (31.3 $\pm 7.21 \%$; $\mathrm{p}<0.05$; Figure 4B). The density of the regenerated tissue was higher in the TSAH/rhBMP-2 group than in the other groups ( $p<0.05$; Figure 4C).

Sequential fluorescence labelling histomorphometric analysis

The regions above the labial cortical bone were chosen to analyse the fluorescence labelling and assess the mineralisation process (Figure 5 ). The results showed the shape of tetracycline hydrochloride (TE) labelling (yellow) matched the linear fibre tissue in both the TSAH and TSAH/ rhBMP-2 groups at week 3. Next, alizarin red ( $A L)$ labelling (red) was distributed around the TE labelling at week 6. Finally, calcein (CA) labelling (green) was detected clearly in both groups until week 9, indicating that mineralisation was occurring. There was a trend for areas of TE and AL labelling in the TSAH group to be weaker than those in the TSAH/rhBMP-2 group in the first 6 weeks. However, at week 9, the areas of CA labelling were comparable. These results demonstrated that TSAH alone can induce calcium deposition.

\section{Histological evaluation}

No inflammatory cells were found at 4, 8, or 12 weeks in any group from decalcified sections stained with HE (Figure 6A-D). There was no evidence of an immune response in any group. Multinucleated giant cells were found adjacent to the remnant hydrogel, which may participate in the degradation of TSAH. The degradation rates of TSAH in groups TSAH and TSAH/rhBMP-2 increased gradually from weeks 4 to 12 . However, at 12 weeks, some TSAH was not degraded and remained at the centre of the implantation areas. Nevertheless, some cells, such as osteoblasts and chondrocytes, were found around the remnant hydrogel at 4 weeks postsurgery. Moreover, in some areas, osteoid structures were observed at 12 weeks post-surgery in the peripheral regions of the material in the TSAH and TSAH/rhBMP-2 groups. In the CPC/rhBMP-2 control group very little mineralised tissue was observed in the transplant areas at 12 weeks, consistent with the radiographic and micro-CT results. The normal anatomical structure of intact bone and the periosteum were seen in the PBS group.

Immunohistochemical staining of osteoblast differentiation markers is shown in Figures 6E-F (OPN) and 6G-H (OCN). Newly mineralised tissue showed high expression of OPN in weeks 4-12 and weak immunostaining for OCN in the TSAH and TSAH/rhBMP-2 groups.
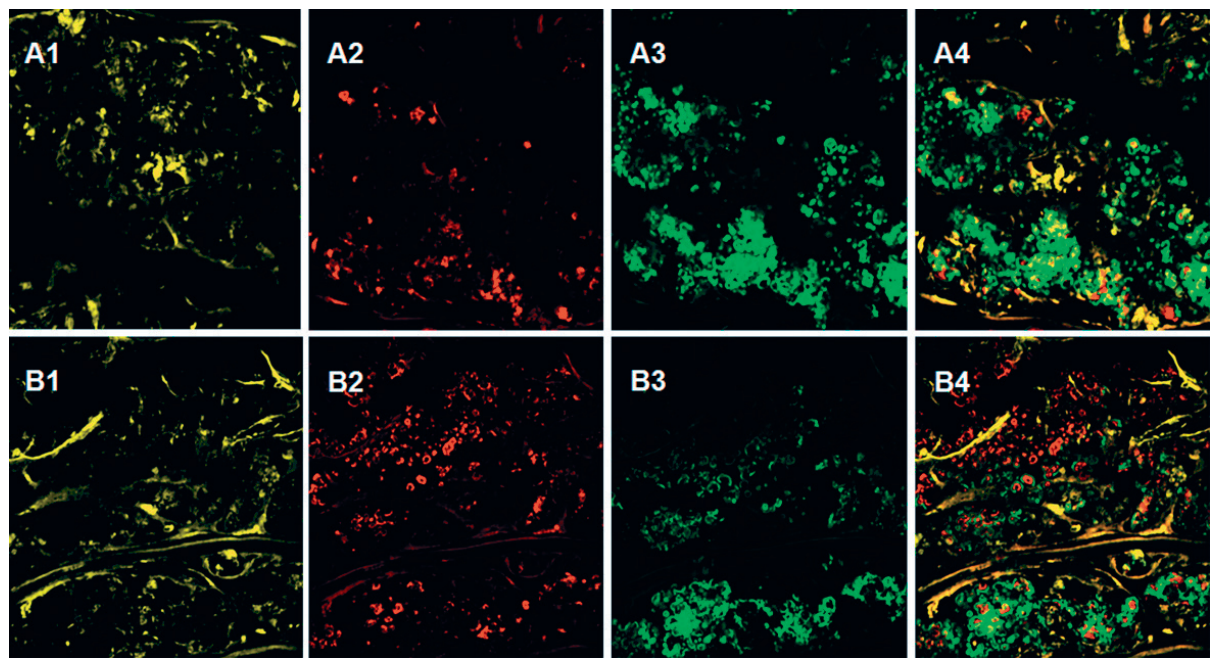

Figure 5- Tetracycline hydrochloride (TE) labelling at 3 weeks (yellow, A1, B1), alizarin red (AL) labelling at 6 weeks (red, $A 2, B 2$ ), calcein (CA) labelling at 9 weeks (green, A3, B3), and the combination of the three types of labelling (A4, B4) in the various groups are shown 


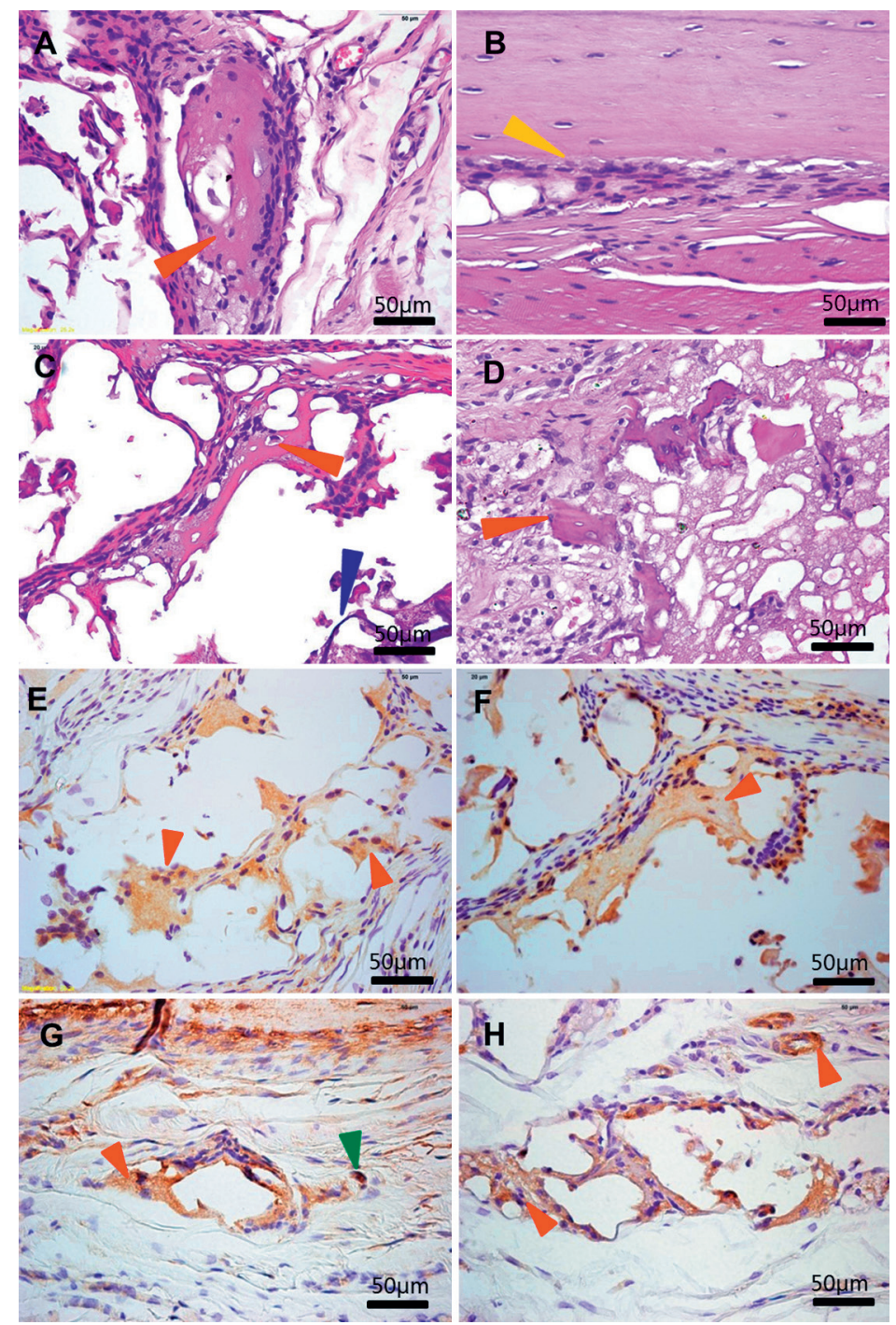

Figure 6- Histological photomicrographs of various groups. Hematoxylin and eosin staining of sections in the thermosensitive alginate hydrogel (TSAH) group (A), phosphate buffer solution (PBS) negative control group (B), TSAH/bone morphogenetic protein-2 (BMP-2) group (C) and calcium phosphate cement (CPC)/BMP-2 control group (D) at week 12 post-surgery. The red arrows $(A, C)$ indicate doubtful chondrocytes and osteoblasts. The blue arrow $(C)$ indicates remnant hydrogel. The yellow arrow (B) indicates intact bone and periosteum. The orange arrow (D) indicates osteoid tissue. Osteopontin (OPN) immunohistochemical staining of sections in the TSAH (E) and TSAH/BMP-2 groups (F) at 12 weeks post-surgery. The red arrows indicate OPN-specific stained cells. Osteocalcin (OCN) immunohistochemical staining of sections in the TSAH (G) and TSAH/BMP-2 groups $(\mathrm{H})$ at 12 weeks post-surgery. The red arrows indicate OCN-specific stained cells. The green arrow indicates dot-like OCN-specific staining

\section{DISCUSSION}

Bone augmentation techniques have recently developed in a noticeable manner. Subperiosteal tunnelling injection is a minimally invasive technique for bone regeneration. Stevens, et al. ${ }^{21}$ (2005) reported the use of such technique to obtain new bone tissue ${ }^{21}$. However, due to the poor plasticity of most of the injectable bone substitute materials ${ }^{10,14}$ and their diffusion after injection in vivo ${ }^{6}$, the technique has not been used widely.

The sol-gel transformation of the TSAH prepared here occurred at $37^{\circ} \mathrm{C}$. When the flowable material was injected through a syringe needle into tissue, under the effect of body temperature, in situ it turned into a gel form and was stable on the bone surface. It was strong enough to support the mucoperiosteum effectively without leakage into the surrounding tissues like the CPC. CPC can be washed out by surgical bleeding after injection into the subperiosteum. This highlights the importance of choosing an appropriate protein factor carrier. It also showed that the TSAH material could meet the operating requirements.

In bone tissue engineering, the degradation rate of the scaffold should be consistent with the new 
bone formation rate ${ }^{17}$. Previous research showed that the degradation time of TSAH can be controlled. With $29 \%$ PNIPAAm grafting, $40 \%$ of the TSAH was degraded in 28 days, matching the bone formation rate ${ }^{23}$.

RhBMP-2 can effectively improve the bone formation and many materials have been reported as carriers for it in animal and clinical studies ${ }^{4,11}$. Alginate hydrogel can retain rhBMP-2 through an electrostatic interaction with the positively charged protein ${ }^{19}$. As a scaffold for protein growth factors, TSAH can provide sustained release of rhBMP-2 and maintain its bioactivity. The rhBMP-2 released from the TSAH into the medium increased the ALP activity of hBMSCs from day 3 to day 15 .

Regarding the osteogenic capabilities of TSAH in vivo, micro-CT showed that both the TSAH and TSAH/rhBMP-2 groups had high-density masses in the augmented area. This suggests that, with or without rhBMP-2, the TSAH material itself can induce mineralisation. When combined with rhBMP-2, the density and volume of mineralised tissue increased and osteogenesis could be enhanced.

Fluorescence labelling revealed that calcium deposition began at week 3 in the TSAH and TSAH/rhBMP-2 groups and was deposited continuously throughout the observation period. The fluorescence intensity in both groups at week 9 was enhanced versus that at weeks 3 and 6, suggesting that the mineralisation activity increased with time. The deposition expanded beyond the immediate mineralisation centre. Closer to the bone surface, more calcium was deposited, indicating that attachment to the bone surface improved mineralisation. In the third month, the fluorescence intensity increased, indicating that mineralisation was increasingly active. Prolonging the observation time may lead to detection of more bone tissue formation.

Osteopontin is a non-collagenous bone matrix glycoprotein produced by preosteoblasts and osteoblasts in the early stages of mesenchymal proliferation, pro-mineralization, and mineralization. It is a marker of mature osteoblasts ${ }^{9}$. Osteocalcin is expressed by mature osteoblasts in the late stages of osteogenic differentiation. It is considered the most specific marker of osteogenesis 5 . In this study, immunohistochemistry revealed OPN expression at 4,8 , and 12 weeks post-surgery in both the TSAH and TSAH/rhBMP-2 groups, further indicating that the TSAH material itself can induce mineralisation after contact with the periosteum. There were a few coloured cells after OCN immunohistochemical staining, in which the scattered dot-like specific staining matched the dot-like colouring in bone fluorescence labelling, suggesting that calcium deposition activity in some areas had resulted in the late stage of mineralization. However, there were no large areas of OCN-specific staining after 12 weeks, showing that the osteogenic differentiation of cells in vivo was mostly restricted to the early stage. From specimens at 12 weeks, alginate remained in the centre of the implants. Calcium deposition was located mainly at the edge of the implants. This suggests that the material needs further improvement. Methods could include adding inorganic filler to increase bone conductivity, enhancing nutritional metabolism inside the material, increasing blood supply, and adding further growth factor(s).

\section{CONCLUSIONS}

In this study, an injectable, sol-gel reversible thermo-sensitive alginate hydrogel was developed. With a subperiosteal tunnelling injection technique, this bone substitute was injected under the maxillary labial periosteum, gelled in situ, and finally induced hard tissue formation, to some extent. However, the hard tissue formed was mainly at the early stages of mineralization and predominantly at the periphery of the material. No mature bone tissue was detected. The results suggest the material needs further improvement.

\section{REFERENCES}

1- Boix D, Weiss P, Gauthier O, Guicheux J, Bouler JM, Pilet P, et al. Injectable bone substitute to preserve alveolar ridge resorption after tooth extraction: a study in a dog. J Mater Sci Mater Med. 2006;17(11):1145-52.

2- Boyne PJ, Salina S, Nakamura A, Audia F, Shabahang S. Bone regeneration using rhBMP-2 induction in hemimandibulectomy type defects of elderly sub-human primates. Cell Tissue Bank. 2005; 7(1):1-10.

3- Buck BE, Malinin TI. Human bone and tissue allografts. Preparation and safety. Clin Orthop Relat Res. 1994(303):8-17. 4- Cicciù M, Herford AS, Cicciù D, Tandon R, Maiorana C. Recombinant human bone morphogenetic protein-2 promote and stabilize hard and soft tissue healing for large mandibular new bone reconstruction defects. J Craniofac Surg. 2014;25(3):860-2. 5- Cowles EA, DeRome ME, Pastizzo G, Brailey LL, Gronowicz GA. Mineralization and the expression of matrix proteins during in vivo bone development. Calcif Tissue Int. 1998;62(1):74-82.

6- Driessens FC, Boltong MG, Bermudes O, Planell JA, Ginebra MP, Fernández E. Effective formulation for the preparation of calcium phosphate bone cements. J Mater Sci Mater Med. 1994;5(3):16470.

7- Gauthier O, Bouler JM, Weiss P, Bosco J, Daculsi G, Aguado E. Kinetic study of bone ingrowth and ceramic resorption associated with the implantation of different injectable calcium-phosphate bone substitutes. J Biomed Mater Res, 1999;47(1):28-35.

8- Gazdag AR, Lane JM, Glaser D, Forster RA. Alternatives to autogenous bone graft: efficacy and indications. J Am Acad Orthop Surg. 1995;3(1):1-8.

9- Giachelli CM, Steitz S. Osteopontin: a versatile regulator of inflammation and biomineralization. Matrix Biol. 2000;19(7):61522.

10- Hjorting-Hansen E, Worsaae N, Lemons JE. Histologic response after implantation of porous hydroxylapatite ceramic in humans. Int J Oral Maxillofac Implants. 1990;5(3):255-63. 
11- Jiang X, Zhao J, Wang S, Sun X, Zhang X, Chen J, et al. Mandibular repair in rats with premineralized silk scaffolds and BMP-2-modified bMSCs. Biomaterials. 2009;30(27):4522-32.

12- Kent JN, Quinn JH, Zide MF, Finger IM, Jarcho M, Rothstein SS. Correction of alveolar ridge deficiencies with nonresorbable hydroxylapatite. J Am Dent Assoc. 1982;105(6):993-1001.

13- Kent JN, Quinn JH, Zide MF, Guerra LR, Boyne PJ. Alveolar ridge augmentation using nonresorbable hydroxylapatite with or without autogenous cancellous bone. J Oral Maxillofac Surg. $1983 ; 41(10): 629-42$.

14- Kim J, Kim IS, Cho TH, Lee KB, Hwang SJ, Tae G, et al. Bone regeneration using hyaluronic acid-based hydrogel with bone morphogenic protein-2 and human mesenchymal stem cells. Biomaterials. 2007;28(10):1830-7.

15- Kolambkar YM, Dupont KM, Boerckel JD, Huebsch N, Mooney DJ, Hutmacher DW, et al. An alginate-based hybrid system for growth factor delivery in the functional repair of large bone defects. Biomaterials. 2011;32(1):65-74.

16- Kretlow JD, Young S, Klouda L, Wong M, Mikos AG. Injectable biomaterials for regenerating complex craniofacial tissues. Adv Mater. 2009;21(32-33):3368-93.

17- Larsson S, Hannink G. Injectable bone-graft substitutes: current products, their characteristics and indications, and new developments. Injury. 2011;42:S30-4.

18- Lisignoli G, Zini N, Remiddi G, Piacentini A, Puggioli A, Trimarchi $C$, et al. Basic fibroblast growth factor enhances in vitro mineralization of rat bone marrow stromal cells grown on non-woven hyaluronic acid based polymer scaffold. Biomaterials. 2001;22(15):2095-105.
19- Luca L, Rougemont AL, Walpoth BH, Gurny R, Jordan O. The effects of carrier nature and $\mathrm{pH}$ on rhBMP-2-induced ectopic bone formation. J Control Release. 2010;147(1):38-44.

20- Marijnissen WJ, van Osch GJ, Aigner J, van der Veen SW, Hollander AP, Verwoerd-Verhoef $\mathrm{HL}$, et al. Alginate as a chondrocyte-delivery substance in combination with a nonwoven scaffold for cartilage tissue engineering. Biomaterials, 2002;23(6):1511-7.

21- Stevens MM, Marini RP, Schaefer D, Aronson J, Langer R, Shastri VP. In vivo engineering of organs: the bone bioreactor. Proc Natl Acad Sci U S A. 2005;102(32):11450-5.

22- Tan HP, Ramirez CM, Miljkovic N, Li H, Rubin JP, Marra KG. Thermosensitive injectable hyaluronic acid hydrogel for adipose tissue engineering. Biomaterials. 2009;30(36):6844-53.

23- Tan RW, She ZD, Wang MB, Fang Z, Liu YS, Feng QL. Thermosensitive alginate-based injectable hydrogel for tissue engineering. Carbohydr Polym. 2012;87(2):1515-21.

24- Von Arx T, Hardt N, Wallkamm B. The TIME technique: a new method for localized alveolar ridge augmentation prior to placement of dental implants. Int J Oral Maxillofac Implants. 1996;11(3):387-94.

25- Xiao YT, Xiang LX, Shao JZ. Bone morphogenetic protein. Biochem Biophys Res Commun. 2007;362(3):550-3.

26- Zernik J, Twarog K, Upholt WB. Regulation of alkaline phosphatase and alpha 2 (I) procollagen synthesis during early intramembranous bone formation in the rat mandible. Differentiation. 1990;44(3):207-15. 